\title{
From genome editing to blastocyst complementation: A new horizon in heart transplantation?
}

\author{
Igor E. Konstantinov, MD, PhD, FRACS, ${ }^{\mathrm{a}, \mathrm{b}, \mathrm{c}, \mathrm{d}}$ Gregory King, MD, ${ }^{\mathrm{a}, \mathrm{b}, \mathrm{c}}$ and Enzo R. Porrello, PhD ${ }^{\mathrm{c}, \mathrm{d}, \mathrm{e}}$
}

Heart transplantation remains the definitive therapy for endstage heart failure; however, widespread access to transplantation is limited by donor shortages. ${ }^{1}$ In addition to the increasing burden of heart failure secondary to dietary and lifestyle factors, ${ }^{2}$ there is a growing population of patients with congenital heart disease, including those with prior univentricular repair. ${ }^{3}$ As such, alternative strategies for treatment of end-stage heart failure are required. Recent breakthroughs in stem cell medicine and genome editing have led to unprecedented research activity in the field of myocardial regeneration. ${ }^{4,5}$ Generating specialized therapeutic cells, tissue grafts, and even entire organs from stem cells appears to be within our grasp. As such, we are at the cutting edge of what previously seemed impossible, and we will be pushing into unchartered territory as we seek pioneering innovations.

\section{INDUCED PLURIPOTENT STEM CELLS}

The field of stem cell medicine has been revolutionized by the discovery of induced pluripotent stem cells (iPSCs). In 1962, John B. Gurdon ${ }^{6}$ cloned a functional tadpole from the nucleus of a specialized intestinal cell of a frog, demonstrating that the nucleus of a mature cell could be returned to a pluripotent state. In 2006, Yamanaka and Takahashi ${ }^{4}$ discovered 4 transcription factors that can reprogram mature cells into pluripotent stem cells. The resulting iPSCs can be generated from human cells and are capable of differentiating into cell types of all 3 germ layers. ${ }^{7,8}$ In 2012, Gurdon and Yamanaka were jointly awarded The Nobel Prize in Physiology or Medicine. Despite their enormous potential in the field of regenerative medicine, numerous obstacles need to be addressed before iPSCs

\footnotetext{
From the ${ }^{a}$ Department of Cardiac Surgery, Royal Children's Hospital, Melbourne, Victoria, Australia; Departments of ${ }^{\mathrm{b}}$ Paediatrics and ${ }^{\mathrm{e}}$ Anatomy and Physiology, The University of Melbourne, Melbourne, Victoria, Australia; ${ }^{\mathrm{c}}$ Murdoch Children's Research Institute, Melbourne, Victoria, Australia; and ${ }^{\mathrm{d}}$ Melbourne Centre for Cardiovascular Genomics and Regenerative Medicine, Melbourne, Victoria, Australia.

Received for publication Nov 12, 2021; accepted for publication Jan 12, 2022; available ahead of print Jan 21, 2022.

Address for reprints: Igor E. Konstantinov, MD, PhD, FRACS, Department of Cardiac Surgery, Royal Children's Hospital, Flemington Rd, Parkville, VIC 3052, Australia (E-mail: igor.konstantinov@rch.org.au).

JTCVS Techniques 2022;12:177-84

2666-2507

Crown Copyright $(C) 2022$ Published by Elsevier Inc. on behalf of The American Association for Thoracic Surgery. This is an open access article under the CC BY-NCND license (http://creativecommons.org/licenses/by-nc-nd/4.0/).

https://doi.org/10.1016/j.xjtc.2022.01.012
}

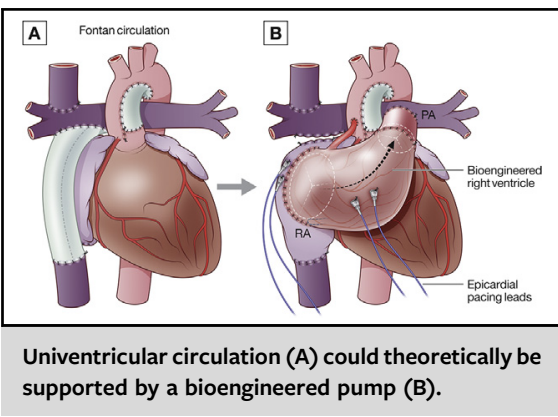

CENTRAL MESSAGE

Rapid advances in stem cell medicine and genome editing technology may result in a paradigm shift in heart transplantation.

can be widely utilized in clinical practice. In particular, the potential for tumorgenicity, immunogenicity, and the integration of transplanted cells are of concern. ${ }^{9}$ Nonetheless, Yamanaka's discovery laid the foundation from which a new frontier in transplantation can be explored.

\section{GENOME EDITING}

In combination with the progress in stem cell research, recent advances in the field of genome editing may have significant implications for transplantation. Namely, the discovery of clustered regularly interspaced short palindromic repeats (CRISPR), and CRISPR-associated 9 (Cas9) protein, has transformed the study of molecular life sciences. Derived from ancient bacterial immune pathways, CRISPR-Cas9 technology functions as a form of a molecular machinery that allows editing of virtually any genome sequence. ${ }^{5}$ For their work in discovering CRISPR-Cas9 as a tool for genome editing, Jennifer A. Doudna and Emmanuelle Charpentier were jointly awarded the 2020 Nobel Prize in Chemistry. Although CRISPRCas9 is a hugely promising technology, several challenges still face its widespread clinical applications. Most importantly, unintended on-target genome damage, as well as off-target effects, risk introducing pathogenic mutations into edited cells. ${ }^{10,11}$ Such mutations could become neoplastic with time, particularly in iPSCs, given their long replicative lifespan. As such, comprehensive methods for screening and detecting genome damage are mandatory 
before patient transplantation of edited cells and their derived products. ${ }^{10,11}$

\section{STEM CELL DONORS}

Immune-mediated rejection is a critical obstacle in the widespread use of iPSCs and the potential grafts that may be derived from them. ${ }^{9}$ A source of donor iPSCs with no (autologous) or reduced (universal donor-derived or genome-edited) immunogenicity is the crucial first step for bioengineered organs in vitro and in vivo.

\section{Autologous iPSCs}

iPSCs created from a patient's own cells provide the incredible possibility of autologous graft and organ transplantation. Despite initial controversies, it is now established that autologous iPSCs do provide the opportunity to develop rejection-free cell therapies. ${ }^{12-15}$ However, the cost per individual patient and the time required to generate sufficient cell quantities or develop grafts for transplantation is currently seen as a prohibitive factor in the utilization of autologous iPSCs. ${ }^{16,17}$ This is particularly problematic for time-sensitive clinical indications, such as ischemic cardiomyopathy. However, in conditions where the need for transplantation may be foreshadowed several years in advance, such as hypoplastic left heart syndrome, autologous iPSCs offer the prospect of tissue grafts or entire heart transplantation, without the need for lifelong immunosuppression.

\section{Universal Donor iPSCs}

In hematopoietic stem cell and solid organ transplantation, immune-mediated rejection is mitigated by matching of donor and recipient human leukocyte antigen (HLA) haplotypes. However, given the variety of HLA haplotypes, the logistics required to prepare the necessary quantity of iPSC lines is currently not practical. Alternatively, it has been proposed that iPSC lines derived from donors who are homozygote for common HLA types could cover a substantial proportion of the population. Such an approach would still require significant logistical hurdles to be overcome and would likely be dependent on large-scale collaboration between existing biobanks. For instance, it has been estimated that to establish a biobank of iPSCs that would cover $90 \%$ of the Japanese population, 140 unique HLA-homozygous donors would be needed, which would require typing of $\sim 160,000$ individuals. ${ }^{18}$ For societies with a broader range of ethnicities, this number would increase. ${ }^{19,20}$

\section{Genome-Edited iPSCs}

To supplement biobanking of HLA-homozygous donors, a recent study utilized genome-editing technology to develop pseudohomozygous iPSCs by HLA allele-specific editing of HLA heterozygote donors. ${ }^{21}$ Although a previous primate study demonstrated a reduced immune response to
HLA-matched allogenic iPSC-cardiomyocytes, immunosuppression is still likely to be required, albeit for a shorter duration and at a lower dose. ${ }^{22}$ This is in part due to the wide variety of minor histocompatibility antigens that are expressed in the cleft of major histocompatibility complex (MHC) antigens, and differ between donor and recipient, even in the case of HLA matching. ${ }^{23}$

Furthermore, genome-editing technology can be used to delete the classical class I HLA (HLA-A, HLA-B, and HLA-C), either by direct exon targeting or by interfering with the $\beta 2$-microglobulin gene ( $\beta 2$-microglobulin forms a heterodimer with class I HLA and is necessary for class-I HLA presentation on the cell surface) (Figure 1, A). With deletion of classical class I HLA, the iPSC may avoid cytotoxic T-cells. However, with complete absence of class I HLA the iPSC will be targeted by natural killer (NK) cells, through the missing self-response. Moreover, deletion of all classical class-I HLA renders a cell incapable of antigen presentation and risks uncontrolled proliferation of cells that are tumorogenic or infected by pathogens. Rather, targeted deletion of HLA-A and HLA-B (Figure 1, B), with retention of a single HLA-C allele, can create iPSCs that reduce both NK cell and cytotoxic T-cell destruction, because HLA-C presentation can also inhibit NK cell activity. ${ }^{21}$ Furthermore, HLA-C retention provides some capacity for antigen presentation, reducing the risks related to tumorigenesis and infection. ${ }^{21}$ Of note, given the relative lack of diversity in the HLA-C haplotype, it is estimated that only 12 donors with various HLA-C haplotypes would be required to provide cells that could create iPSCs for most of the world's population. ${ }^{21}$ Moreover, to overcome NK cell destruction, genome editing can overexpress a single-chain HLA-E/ $\beta 2$ microglobulin fusion, in which the nonclassical HLA-E can inhibit $\mathrm{NK}$ cell activity to a degree. ${ }^{24}$ In addition, the class-II MHC transactivator gene can be disrupted, depleting class-II MHC and, thus, avoiding HLA-DRactivated helper T-cell toxicity. ${ }^{21}$ Immune cloaking via genome editing, to induce expression of immune checkpoints molecules, is another strategy to inhibit immunemediated rejection of allogenic iPSCs. ${ }^{25}$

\section{CLINICAL APPLICATIONS}

The potential clinical applications of iPSCs ranges from cellular therapy to the production of entire organs for transplantation. To date, preclinical and clinical studies have predominantly focused on cellular therapies; however, there has been substantial progress in the pursuit of bioengineered cardiac tissues and organs suitable for transplantation.

\section{Cellular Therapy}

Previous animal studies, using bone marrow-derived cells injected into myocardial peri-infarct areas, 


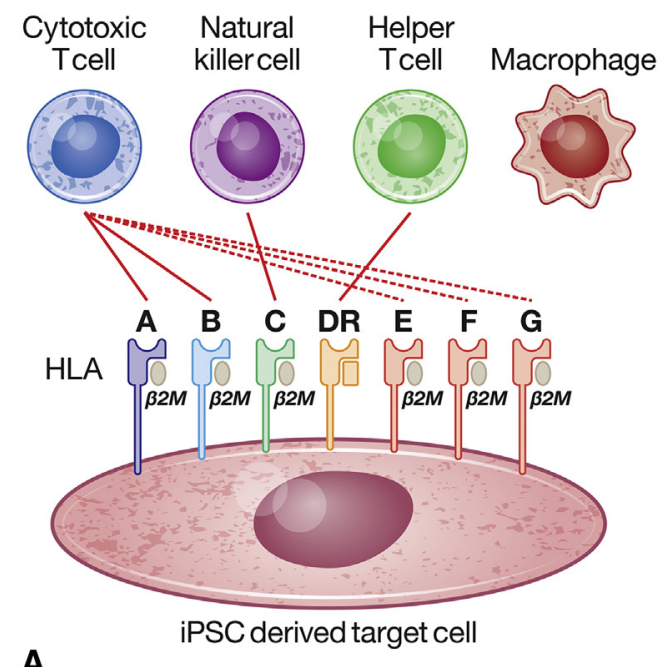

A

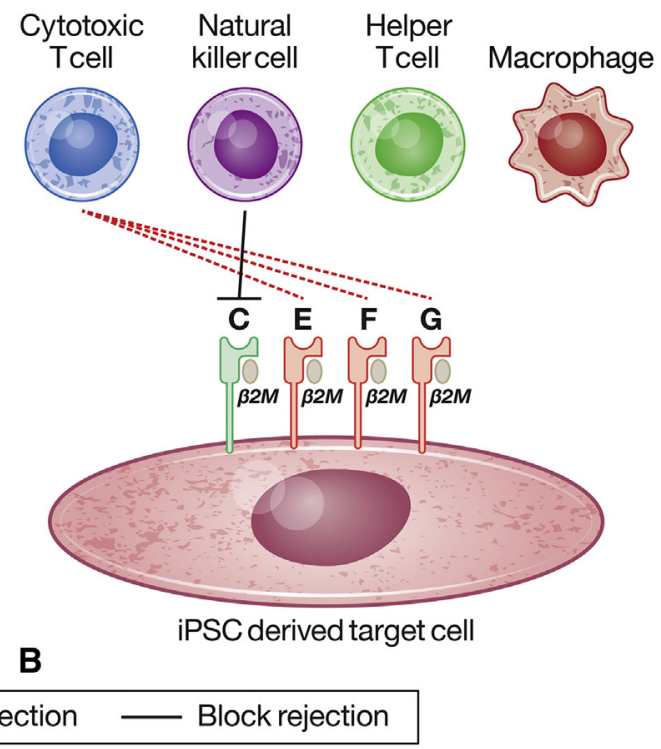

FIGURE 1. Genome editing of induced pluripotent stem cells (iPSCs) can reduce the risk of immune mediated rejection, supplementing a biobank of universal donor cells, tissues, and organs for use in transplantation. A, Rejection of an allogenic iPSC or its derived tissues is mediated through human leukocyte antigen (HLA) mismatching. Helper T-cell (Th) activation, via class-II major histocompatibility complex (MHC) molecules, namely HLA-DR, leads to cytokine release and recruitment of various immune cells. Cytotoxic T-cell (Tc) and natural killer (NK) cell activation, via classical class-I MHC molecules, namely HLA-A, HLA-B, and HLA-C, lead to destruction of the allogenic iPSC. However, a complete lack of class I MHC molecules (ie, through interference with the $\beta 2$-microglobulin $[\beta 2 \mathrm{M}]$ gene) will lead to NK cell activation through the missing self response. B, Genome editing can create iPSCs with selective depletion of HLA-A, HLA-B, and HLA-DR, while retaining HLA-C, which can protect against Tc, Th, and NK mediated destruction. Nonetheless, cell depletion can still occur through minor histocompatibility antigens, expressed in the cleft of MHC molecules, and mismatching of nonclassical MHC molecules (such as HLA-E, HLA-F, and HLA-G).

demonstrated an improvement in cardiac function and regeneration of cardiomyocytes. ${ }^{26}$ Although these results generated enormous excitement in the field of cardiac regenerative medicine, they were not always reproducible. ${ }^{27}$ Subsequent studies have raised doubt about both the efficacy and mechanism of adult stem cell therapies for cardiac disease. ${ }^{28-30}$ In a recent study, intramyocardial injection of live or dead stem cells, or a chemical inducer of innate immunity, all resulted in activation of an innate immune response and improvement in cardiac function. ${ }^{31}$ As such, the beneficial effects of adult stem cell therapies may be due to activation of the innate immune response, rather than stem cell engraftment and differentiation into cardiomyocytes. Despite the controversies surrounding adult stem cell therapies, ${ }^{28}$ there are ongoing preclinical and clinical studies investigating the potential benefits of iPSC-derived cardiomyocytes as a form of injectable cellular therapy. ${ }^{32-34}$ Industrial-scale production of human cardiomyocytes from pluripotent stem cells is now possible, ${ }^{35}$ enabling transplantation of clinically relevant numbers of cardiomyocytes (up to 1 billion) following myocardial infarction in a nonhuman primate model. ${ }^{36} \mathrm{~A}$ number of clinical trials involving direct injection of PSC-derived cardiomyocytes in adults with end-stage heart failure are now underway although some safety concerns, particularly arrhythmias, have been documented in preclinical animal studies. ${ }^{36,37}$

\section{Bioengineering In Vitro}

Cardiac tissue. Human iPSCs can be differentiated into the 4 major cell types of the heart (ie, cardiomyocytes, endothelial cells, cardiac fibroblasts, and smooth muscle cells) and used for cardiac tissue engineering in vitro. ${ }^{38-40}$ Although current protocols for differentiating iPSCs can reliably produce high-yield and high-purity cardiomyocytes, ${ }^{41,42}$ achieving sufficient maturation of these cells to facilitate optimal electrical and mechanical continuum remains difficult. Nonetheless, various strategies, including long-term culture, ${ }^{43}$ co-culture with supportive cell types, ${ }^{44}$ and electrical ${ }^{38}$ and mechanical stimulation ${ }^{45}$ have led to improved maturation of cardiomyocytes. In addition to cellular maturity, replicating the anisotropy between and within native cardiomyocytes is necessary to optimize function and prevent maladaptive processes in bioengineered cardiac tissue. ${ }^{40}$ To achieve this, various bioengineering methods and materials have been utilized, ranging from cyclic stretch ${ }^{46}$ to fabricated replicates of the native extracellular matrix (with both natural and synthetic polymers), ${ }^{47,48}$ hydrogels, ${ }^{49}$ and more advanced techniques such as melt electrospinning writing. ${ }^{50}$ In animal studies, bioengineered 
cardiac tissue created from iPSCs have demonstrated evidence of engraftment, vascularization, and electrical coupling to the recipient cardiac tissue, as well as an improvement in cardiac function. ${ }^{51,52}$ Furthermore, successful epicardial transplantation of human scale (up to $5 \mathrm{~cm} \times 7 \mathrm{~cm}$ ) patches in porcine hearts has demonstrated safety, feasibility, and efficacy of such an approach. ${ }^{52,53}$ Another study demonstrated increased retention and engraftment of intramyocardially injected cardiomyocytes, when delivered concomitantly with epicardial transplantation of a human mesenchymal stem cell-loaded patch, which provided a complimentary microenvironment and enhanced vascular regeneration. ${ }^{54}$ Despite the progress in bioengineering cardiac tissue, ongoing challenges related to integration, compatibility, sustained viability, and electromechanical coupling with native myocardium remain before successful clinical translation. ${ }^{40}$

Cardiac pump. Progress in bioengineering cardiac tissue has meant the prospect of a bioengineered cardiac pump suitable for transplantation is becoming increasingly realistic. However, generating cardiac tissue that is thick enough to withstand the loading forces of the heart and produce sufficient contractile power to supplement or substitute ventricular function remains difficult. In particular, generating a physiological cell density of cardiomyocytes $\left(\sim 10^{8}\right.$ cells $\left./ \mathrm{cm}^{3}\right)$ while replicating the geometry of cardiac structures and integrating a vascular network (Figure 2) to ensure viability of thick bioengineered cardiac tissue is challenging. ${ }^{40,55-57}$ Several approaches exist for tissue vascularization, including natural ingrowth of blood vessels from the host ${ }^{58,59}$ and developing neovascularization in the tissue before transplantation. ${ }^{52,60}$ Unfortunately, both techniques often result in disorganized vascular networks, not suitable for functional myocardium. ${ }^{40} \mathrm{~A}$ more promising vascularization technique is 3-dimensional (3D) bioprinting and micropatterning, ${ }^{47}$ which result in a more controllable vascular network suitable for functional myocardium. Recently, researchers used 3D bioprinting to bioengineer personalized, thick, perfusable cardiac patches derived from iPSCs. ${ }^{61}$ Fabrication techniques for producing cardiac structures, whether it be ventricle-like structures or indeed whole hearts, are divided into top-down (seeding of cells within an acellular preformed scaffold) and bottom-up (modular assembly of cellular building blocks) strategies. ${ }^{40,57}$ Top-down fabrication using decellularized whole hearts that were reseeded with cardiomyocytes, or multipotent cardiovascular progenitors, and perfused with vascular conduits, have demonstrated evidence of nascent pump function. ${ }^{62-64}$ Furthermore, a recent study used iPSCs encapsulated in a native extracellular matrix-based hydrogel to 3D bioprint a perfusable, electromechanically functional 3D-chambered structure. ${ }^{65}$ Although the pinnacle of bioengineering would be to create entire organs that are readily available for transplantation, the more imminent

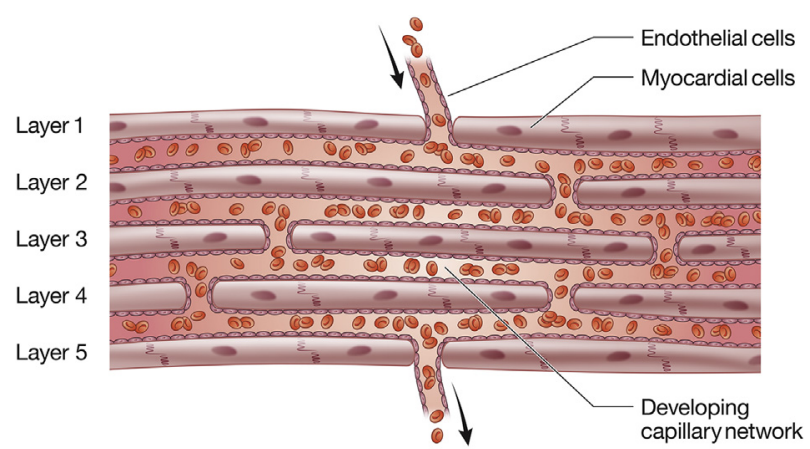

FIGURE 2. Induced pluripotent stem cells (iPSCs) can be differentiated into cardiomyocytes and endothelial cells to be used to bioengineer vascularized cardiac tissue. iPSCs, either autologous or sourced from biobanks of universal donors or genome edited cell lines, can be used to generate a vascularized cardiac patch for use in transplantation.

clinical application of bioengineered cardiac tissue could come in the form of a bioengineered pump. In as much as current contractile chambers begin to display signs of future potential, much work is yet to be done to create a bioengineered cardiac pump capable of meaningful clinical translation. In all likelihood, the first cardiac pumps, if ever attainable, would provide support for the lowpressure pulmonary circulation.

For example, given the growing number of patients with congenital heart disease and prior univentricular repair, the initial clinical translation of such technology could be in supporting the low-pressure pulmonary circulation in patients following Fontan operation or other univentricular palliations (Figure 3). In such patients decreasing central venous pressure by only $10 \mathrm{~mm} \mathrm{Hg}$ may result in resolution of Fontan circulation failure. To replicate cardiac structures with complex geometry, such as valves, bioengineered or homograft prostheses could be used.

\section{Bioengineering In Vivo}

Breakthroughs in genome editing and stem cell biology have also opened the possibility of creating organs from human iPSCs through blastocyst complementation (Figure 4). In this process, a blastocyst from 1 organism is genetically modified so that it lacks the necessary lineage to develop the tissue of interest. ${ }^{66}$ Human iPSCs can then be microinjected into the modified blastocyst, where they would hold a competitive advantage because the host blastocyst will be incapable of specific organogenesis. ${ }^{67}$ The modified blastocyst is then transferred into a maternal surrogate. A seminal study delivered mouse embryonic cells to a rat blastocyst deficient of Pdx1, which is required for pancreas development. ${ }^{68}$ Ultimately, a rat-sized pancreas derived of mouse embryonic stem cells was developed. However, the heart appears to be less amenable, if at all, to blastocyst complementation. Thus, due to the lack of a single mutation that 


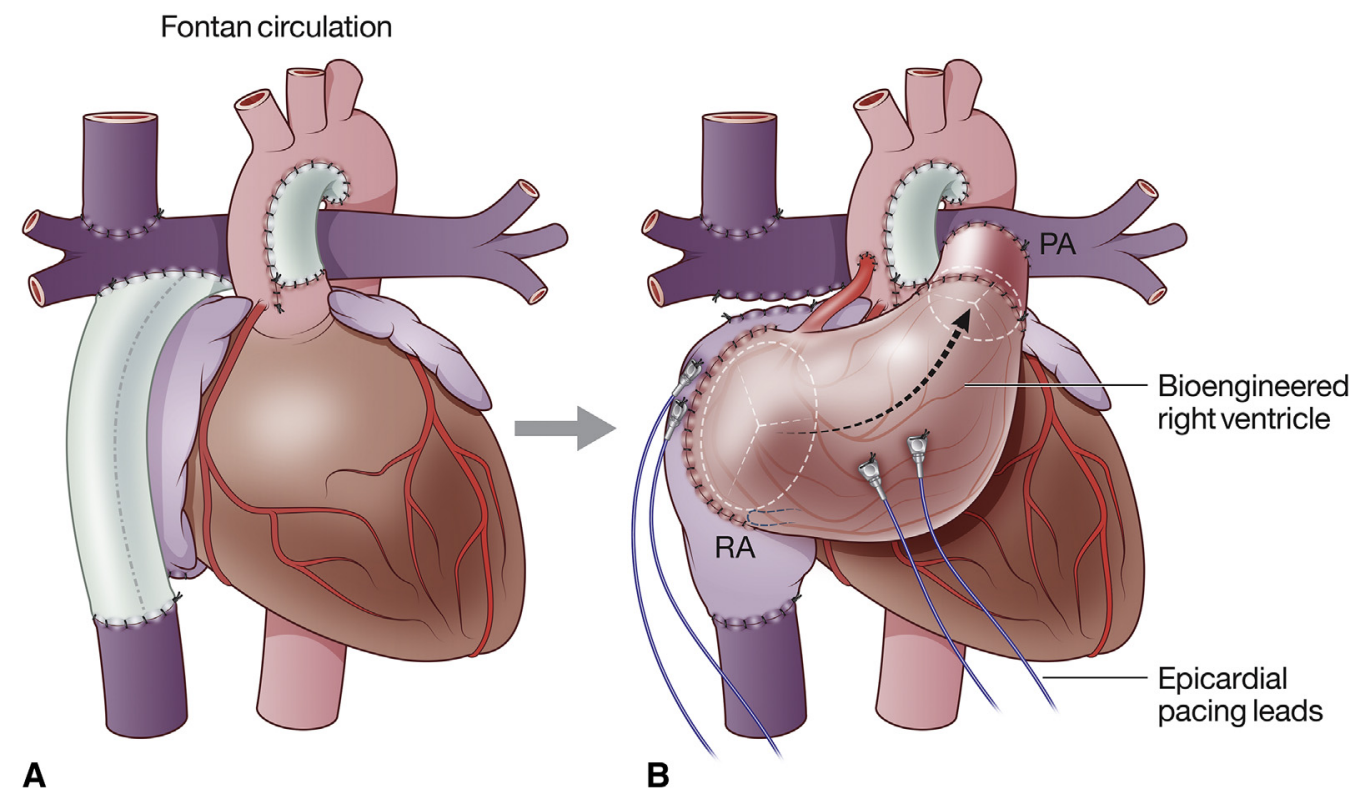

FIGURE 3. The potential clinical translation of a bioengineered pump into a patient with prior univentricular repair. A, Anatomical representation of an extracardiac Fontan circulation in a patient with hypoplastic left heart syndrome and previous Norwood repair. Many manifestations of a failing Fontan circulation, such as protein losing enteropathy, are related to raised central venous pressure (CVP), due to the lack of a sub-pulmonic ventricle. B, The first clinical translation of a bioengineered pump may be in the form of right ventricle to support the low-pressure pulmonary circulation in patients after Fontan operation. Lowering CVP, even by a few millimeters of mercury, can have a significant impact on the quality of life of patients with Fontan circulation. Electromechanical synchronization and perfusion of the graft could potentially be achieved by placement of epicardial pacing leads, and anastomosis of bioengineered vessels from the graft to the existing vasculature. $R A$, Right atrium; $P A$, pulmonary artery.

would result in an empty cardiac development niche, the chimera's heart would consist of both host and donor cells. ${ }^{67,69}$ To date, human-animal chimeric embryos have been formed with pigs, cattle, and most recently monkeys, albeit with relatively low human cell contributions to the developing embryo. ${ }^{69,70}$ It should be kept in mind that currently most organs produced by blastocyst complementation are vascularized by host tissues, making them

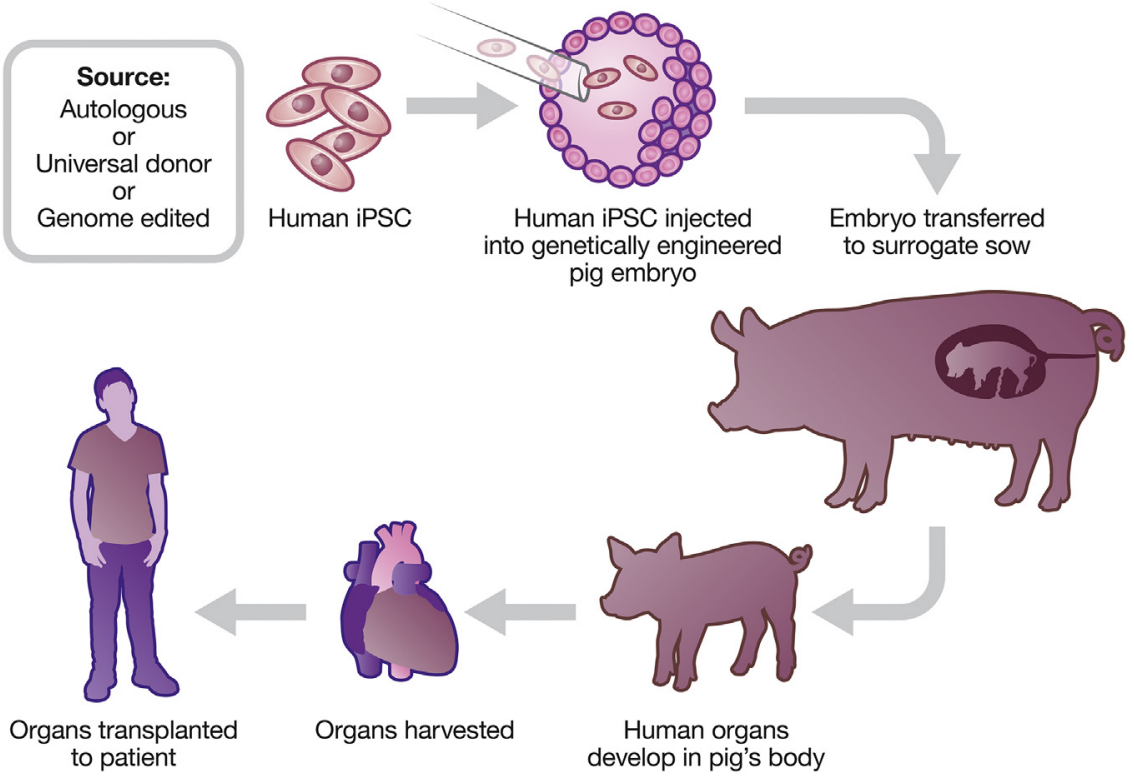

FIGURE 4. Human stem cells could be injected into a pig blastocyst to create a chimeric blastocyst that is then transferred into a surrogate sow to facilitate organogenesis. The cells of the host blastocyst would be edited to be incapable of developing specific organs. As such, within the chimeric blastocyst, the injected induced pluripotent stem cells (iPSCs) would hold a competitive advantage, which would allow organs to develop predominantly derived of human cell lines. The mature humanized organs of chimeric animals could be harvested and used in transplantation. 
unfit for transplantation. Remarkably, a pig chimera with endothelium exclusively derived from donor human iPSCs has been created ${ }^{66}$ Thus, it now appears feasible to produce organs with human vasculature.

Numerous challenges remain before the effective generation of chimeric organs, including overcoming the evolutionary divergence between human donor cells and the animal host environment. ${ }^{71}$ It may seem attractive, for instance, that iPSCs from a child born with hypoplastic left heart syndrome could be utilized for personalized organogenesis and subsequent transplantation. However, it is not clear, even if such a heart could be created, how a different rate of somatic growth in donor and host mammalian species will influence organogenesis and the possibility of personalized transplantation..$^{72}$ Finally, it is important to acknowledge the ethical concerns regarding human-animal chimeras and the potential contribution of human iPSCs to neura ${ }^{73}$ and germ lines. ${ }^{74}$ It is imperative that any research in this area does not ignore these ethical issues. ${ }^{75,76}$ Although it is not clear yet, whether the sustained clinical translation of genome editing to heart transplantation will occur as result of genome edited xenotransplantation ${ }^{77}$ or bioengineered stem cell derived pumps, the impact of genome editing on heart transplantation is likely to be significant.

\section{CONCLUSIONS}

Due to revolutionary advances in the field of stem cell research and genome editing, we are now positioned at the frontier of what previously seemed impossible. iPSCs offer highly personalized cell lines that may form the foundation for bioengineered organs, opening the possibility of transplantation without the need for immunosuppression. It is conceivable that within a few years, bioengineered cardiac pumps will be available for clinical application. Furthermore, with the rapid pace of development in bioengineering, a new era in heart transplantation may appear on the horizon.

\section{Conflict of Interest Statement}

The authors reported no conflicts of interest.

The Journal policy requires editors and reviewers to disclose conflicts of interest and to decline handling or reviewing manuscripts for which they may have a conflict of interest. The editors and reviewers of this article have no conflicts of interest.

\section{References}

1. Dharmavaram N, Hess T, Jaeger H, Smith J, Hermsen J, Murray D. National trends in heart donor usage rates: are we efficiently transplanting more hearts? J Am Heart Assoc. 2021;10:e019655.

2. Virani SS, Alonso A, Benjamin EJ, Bittencourt MS, Callaway CW, Carson AP, et al. Heart disease and stroke statistics 2020 update: a report from the American Heart Association. Circulation. 2020;141:e139-596.
3. Schilling C, Dalziel K, Nunn R, Du Plessis K, Shi WY, Celermajer D, et al. The Fontan epidemic: population projections from the Australia and New Zealand Fontan Registry. Int J Cardiol. 2016;219:14-9.

4. Takahashi K, Yamanaka S. Induction of pluripotent stem cells from mouse embryonic and adult fibroblast cultures by defined factors. Cell. 2006;126: 663-76.

5. Jinek M, Chylinski K, Fonfara I, Hauer M, Doudna JA, Charpentier E. A programmable dual-RNA-guided DNA endonuclease in adaptive bacterial immunity. Science. 2012;337:816-21.

6. Gurdon JB. The developmental capacity of nuclei taken from intestinal epithelium cells of feeding tadpoles. J Embryol Exp Morphol. 1962;10:622-40.

7. Takahashi K, Tanabe K, Ohnuki M, Narita M, Ichisaka T, Tomoda K, et al. Induction of pluripotent stem cells from adult human fibroblasts by defined factors. Cell. 2007;131:861-72.

8. Yu J, Vodyanik MA, Smuga-Otto K, Antosiewicz-Bourget J, Frane JL, Tian S, et al. Induced pluripotent stem cell lines derived from human somatic cells. Science. 2007;318:1917-20.

9. Yamanaka S. Pluripotent stem cell-based cell therapy-promise and challenges. Cell Stem Cell. 2020;27:523-31.

10. Kosicki M, Tomberg K, Bradley A. Repair of double-strand breaks induced by CRISPR-Cas9 leads to large deletions and complex rearrangements. Nat Biotechnol. 2018;36:765-71.

11. Saha K, Sontheimer EJ, Brooks PJ, Dwinell MR, Gersbach CA, Liu DR, et al. The NIH somatic cell genome editing program. Nature. 2021;592:195-204.

12. Deuse T, Hu X, Agbor-Enoh S, Koch M, Spitzer M, Gravina A, et al. De novo mutations in mitochondrial DNA of iPSCs produce immunogenic neoepitopes in mice and humans. Nat Biotechnol. 2019;37:1137-44.

13. Zhao T, Zhang Z-N, Rong Z, Xu Y. Immunogenicity of induced pluripotent stem cells. Nature. 2011;474:212-5.

14. Araki R, Uda M, Hoki Y, Sunayama M, Nakamura M, Ando S, et al. Negligible immunogenicity of terminally differentiated cells derived from induced pluripotent or embryonic stem cells. Nature. 2013;494:100-4.

15. Mandai M, Watanabe A, Kurimoto Y, Hirami Y, Morinaga C, Daimon T, et al. Autologous induced stem-cell-derived retinal cells for macular degeneration. N Engl J Med. 2017;376:1038-46.

16. Chakradhar S. An eye to the future: researchers debate best path for stem cellderived therapies. Nat Med. 2016;22:116-9.

17. Madrid M, Sumen C, Aivio S, Saklayen N. Autologous induced pluripotent stem cell-based cell therapies: promise, progress, and challenges. Curr Protoc. 2021; $1: \mathrm{e} 88$.

18. Okita K, Matsumura Y, Sato Y, Okada A, Morizane A, Okamoto S, et al. A more efficient method to generate integration-free human iPS cells. Nat Methods. 2011;8:409-12.

19. Gourraud PA, Gilson L, Girard M, Peschanski M. The role of human leukocyte antigen matching in the development of multiethnic "haplobank" of induced pluripotent stem cell lines. Stem Cells. 2012;30:180-6.

20. Taylor Craig J, Peacock S, Chaudhry Afzal N, Bradley JA, Bolton Eleanor M. Generating an iPSC Bank for HLA-matched tissue transplantation based on known donor and recipient HLA types. Cell Stem Cell. 2012;11:147-52.

21. Xu H, Wang B, Ono M, Kagita A, Fujii K, Sasakawa N, et al. Targeted Disruption of HLA genes via CRISPR-Cas9 generates iPSCs with enhanced immune compatibility. Cell Stem Cell. 2019;24:566-78.e7.

22. Kawamura T, Miyagawa S, Fukushima S, Maeda A, Kashiyama N, Kawamura A, et al. Cardiomyocytes derived from MHC-homozygous induced pluripotent stem cells exhibit reduced allogeneic immunogenicity in MHC-matched non-human primates. Stem Cell Rep. 2016;6:312-20.

23. Trounson A, Boyd NR, Boyd RL. Toward a universal solution: editing compatibility into pluripotent stem cells. Cell Stem Cell. 2019;24:508-10.

24. Gornalusse GG, Hirata RK, Funk SE, Riolobos L, Lopes VS, Manske G, et al. HLA-E-expressing pluripotent stem cells escape allogeneic responses and lysis by NK cells. Nat Biotechnol. 2017;35:765-72.

25. Rong Z, Wang M, Hu Z, Stradner M, Zhu M, Kong H, et al. An effective approach to prevent immune rejection of human ESC-derived allografts. Cell Stem Cell. 2014; 14:121-30.

26. Orlic D, Kajstura J, Chimenti S, Jakoniuk I, Anderson SM, Li B, et al. Bone marrow cells regenerate infarcted myocardium. Nature. 2001;410:701-5.

27. Balsam LB, Wagers AJ, Christensen JL, Kofidis T, Weissman IL, Robbins RC. Haematopoietic stem cells adopt mature haematopoietic fates in ischaemic myocardium. Nature. 2004;428:668-73.

28. Epstein JA. A time to press reset and regenerate cardiac stem cell biology. JAMA Cardiol. 2019;4:95-6. 
29. Sano S, Sano T, Ishigami S, Ito T. Cardiac stem cell therapy: does a newborn infant's heart have infinite potential for stem cell therapy? J Thorac Cardiovasc Surg. 2022;163:242-7.

30. Gyöngyösi M, Haller PM, Blake DJ, Rendon EM. Meta-analysis of cell therapy studies in heart failure and acute myocardial infarction. Circ Res. 2018;123: $301-8$.

31. Vagnozzi RJ, Maillet M, Sargent MA, Khalil H, Johansen AZ, Schwanekamp JA, et al. An acute immune response underlies the benefit of cardiac stem cell therapy. Nature. 2020;577:405-9.

32. Guan X, Xu W, Zhang H, Wang Q, Yu J, Zhang R, et al. Transplantation of human induced pluripotent stem cell-derived cardiomyocytes improves myocardial function and reverses ventricular remodeling in infarcted rat hearts. Stem Cell Res Ther. 2020;11:73.

33. Peinkofer G, Maass M, Pfannkuche K, Sachinidis A, Baldus S, Hescheler J, et al. Persistence of intramyocardially transplanted murine induced pluripotent stem cell-derived cardiomyocytes from different developmental stages. Stem Cell Res Ther. 2021;12:46.

34. Shiba Y, Gomibuchi T, Seto T, Wada Y, Ichimura H, Tanaka Y, et al. Allogeneic transplantation of iPS cell-derived cardiomyocytes regenerates primate hearts. Nature. 2016;538:388-91.

35. Denning C, Borgdorff V, Crutchley J, Firth KA, George V, Karla S, et al. Cardiomyocytes from human pluripotent stem cells: from laboratory curiosity to industrial biomedical platform. Biochim Biophys Acta. 2016;1863: 1728-48.

36. Chong JJ, Yang X, Don CW, Minami E, Liu Y, Weyers JJ, et al. Human embryonic-stem-cell-derived cardiomyocytes regenerate non-human primate hearts. Nature. 2014;510:273-7.

37. Romagnuolo R, Masoudpour H, Porta-Sánchez A, Qiang B, Barry J, Laskary A, et al. Human embryonic stem cell-derived cardiomyocytes regenerate the infarcted pig heart but induce ventricular tachyarrhythmias. Stem Cell Rep. 2019;12:967-81.

38. Ronaldson-Bouchard K, Ma SP, Yeager K, Chen T, Song L, Sirabella D, et al. Advanced maturation of human cardiac tissue grown from pluripotent stem cells. Nature. 2018:556:239-43.

39. Lowenthal J, Gerecht S. If you build it, they will come. Circ Res. 2020;127:225-8.

40. Yadid M, Oved H, Silberman E, Dvir T. Bioengineering approaches to treat the failing heart: from cell biology to 3D printing. Nat Rev Cardiol. 2022;19: 83-99.

41. Palpant NJ, Pabon L, Friedman CE, Roberts M, Hadland B, Zaunbrecher R, et al. Generating high-purity cardiac and endothelial derivatives from patterned mesoderm using human pluripotent stem cells. Nat Protoc. 2017;12:15-31.

42. Lian X, Zhang J, Azarin SM, Zhu K, Hazeltine LB, Bao X, et al. Directed cardiomyocyte differentiation from human pluripotent stem cells by modulating Wnt/ $\beta$-catenin signaling under fully defined conditions. Nat Protoc. 2013;8:162-75.

43. Lundy SD, Zhu WZ, Regnier M, Laflamme MA. Structural and functional maturation of cardiomyocytes derived from human pluripotent stem cells. Stem Cells Dev. 2013;22:1991-2002.

44. Tiburcy M, Hudson JE, Balfanz P, Schlick S, Meyer T, Chang Liao ML, et al. Defined engineered human myocardium with advanced maturation for applications in heart failure modeling and repair. Circulation. 2017;135:1832-47.

45. Tulloch NL, Muskheli V, Razumova MV, Korte FS, Regnier M, Hauch KD, et al. Growth of engineered human myocardium with mechanical loading and vascular coculture. Circ Res. 2011;109:47-59.

46. Riegler J, Tiburcy M, Ebert A, Tzatzalos E, Raaz U, Abilez OJ, et al. Human engineered heart muscles engraft and survive long term in a rodent myocardial infarction model. Circ Res. 2015;117:720-30.

47. Fleischer S, Shapira A, Feiner R, Dvir T. Modular assembly of thick multifunctional cardiac patches. Proc Natl Acad Sci U S A. 2017;114:1898-903.

48. Capulli AK, MacQueen LA, Sheehy SP, Parker KK. Fibrous scaffolds for building hearts and heart parts. Adv Drug Deliv Rev. 2016;96:83-102.

49. Gao L, Kupfer ME, Jung JP, Yang L, Zhang P, Da Sie Y, et al. Myocardial tissue engineering with cells derived from human-induced pluripotent stem cells and a native-like, high-resolution, 3-dimensionally printed scaffold. Circ Res. 2017; 120:1318-25.

50. Castilho M, Feyen D, Flandes-Iparraguirre M, Hochleitner G, Groll J, Doevendans PAF, et al. Electrospinning writing of poly-hydroxymethylglycolide-co- $\varepsilon$-caprolactone-based scaffolds for cardiac tissue engineering. Adv Healthc Mater. 2017;6:10.

51. Weinberger F, Breckwoldt K, Pecha S, Kelly A, Geertz B, Starbatty J, et al. Cardiac repair in Guinea pigs with human engineered heart tissue from induced pluripotent stem cells. Sci Transl Med. 2016;8:363ra148.
52. Gao L, Gregorich ZR, Zhu W, Mattapally S, Oduk Y, Lou X, et al. Large cardiac muscle patches engineered from human induced-pluripotent stem cell-derived cardiac cells improve recovery from myocardial infarction in swine. Circulation 2018;137:1712-30.

53. Querdel E, Reinsch M, Castro L, Kose D, Bahr A, Reich S, et al. Human engineered heart tissue patches remuscularize the injured heart in a dose-dependent manner. Circulation. 2021;143:1991-2006.

54. Park S-J, Kim RY, Park B-W, Lee S, Choi SW, Park J, et al. Dual stem cell therapy synergistically improves cardiac function and vascular regeneration following myocardial infarction. Nat Commun. 2019;10:3123.

55. MacQueen LA, Sheehy SP, Chantre CO, Zimmerman JF, Pasqualini FS, Liu X et al. A tissue-engineered scale model of the heart ventricle. Nat Biomed Eng. 2018:2:930-41.

56. Taylor DA, Hochman-Mendez C, Sampaio LC. Are we close to bioengineering a human-sized, functional heart? J Thorac Cardiovasc Surg. 2020; 159:1357-60.

57. Fang Y, Sun W, Zhang T, Xiong Z. Recent advances on bioengineering approaches for fabrication of functional engineered cardiac pumps: a review. Biomaterials. 2022:280:121298.

58. Sekine H, Shimizu T, Hobo K, Sekiya S, Yang J, Yamato M, et al. Endothelial cell coculture within tissue-engineered cardiomyocyte sheets enhances neovascularization and improves cardiac function of ischemic hearts. Circulation. 2008;118 S145-52.

59. Dvir T, Kedem A, Ruvinov E, Levy O, Freeman I, Landa N, et al. Prevascularization of cardiac patch on the omentum improves its therapeutic outcome. Proc Natl Acad Sci U S A. 2009;106:14990-5.

60. Cui H, Liu C, Esworthy T, Huang Y, Yu ZX, Zhou X, et al. 4D physiologically adaptable cardiac patch: a 4-month in vivo study for the treatment of myocardial infarction. Sci Adv. 2020;6:eabb5067.

61. Noor N, Shapira A, Edri R, Gal I, Wertheim L, Dvir T. 3D Printing of personalized thick and perfusable cardiac patches and hearts. Adv Sci (Weinh). 2019;6:1900344.

62. Ott HC, Matthiesen TS, Goh SK, Black LD, Kren SM, Netoff TI, et al. Perfusiondecellularized matrix: using nature's platform to engineer a bioartificial heart. Nat Med. 2008;14:213-21.

63. Guyette JP, Charest JM, Mills RW, Jank BJ, Moser PT, Gilpin SE, et al. Bioengineering human myocardium on native extracellular matrix. Circ Res. 2016; 118:56-72.

64. Lu T-Y, Lin B, Kim J, Sullivan M, Tobita K, Salama G, et al. Repopulation of decellularized mouse heart with human induced pluripotent stem cell-derived cardiovascular progenitor cells. Nat Commun. 2013;4:2307.

65. Kupfer ME, Lin W-H, Ravikumar V, Qiu K, Wang L, Gao L, et al. In situ expansion, differentiation, and electromechanical coupling of human cardiac muscle in a 3d bioprinted, chambered organoid. Circ Res. 2020;127:207-24.

66. Matsunari H, Watanabe M, Hasegawa K, Uchikura A, Nakano A, Umeyama K, et al. Compensation of disabled organogeneses in genetically modified pig fetuses by blastocyst complementation. Stem Cell Rep. 2020;14:21-33.

67. Crane AT, Aravalli RN, Asakura A, Grande AW, Krishna VD, Carlson DF, et al Interspecies organogenesis for human transplantation. Cell Transpl. 2019;28: 1091-105.

68. Yamaguchi T, Sato H, Kato-Itoh M, Goto T, Hara H, Mizuno N, et al. Interspecies organogenesis generates autologous functional islets. Nature. 2017;542:191-6.

69. Wu J, Platero-Luengo A, Sakurai M, Sugawara A, Gil MA, Yamauchi T, et al Interspecies chimerism with mammalian pluripotent stem cells. Cell. 2017; 168:473-86.e15.

70. Tan T, Wu J, Si C, Dai S, Zhang Y, Sun N, et al. Chimeric contribution of human extended pluripotent stem cells to monkey embryos ex vivo. Cell. 2021;184 2020-32.e14.

71. Garry DJ, Garry MG. Interspecies chimeras and the generation of humanized organs. Circ Res. 2019;124:23-5.

72. Konstantinov IE, Ye XT, Fricke TA. From cellular senescence to regeneration: a quest for the holy grail for the next generation of surgeons? J Thorac Cardiovasc Surg. 2017;154:953-4.

73. Crane AT, Voth JP, Shen FX, Low WC. Concise review: human-animal neurological chimeras: humanized animals or human cells in an animal? Stem Cells. 2019; 37:444-52.

74. Founta KM, Papanayotou C. In vivo generation of organs by blastocyst complementation: advances and challenges. Int J Stem Cells. October 31, 2021 [Epub ahead of print].

75. Crane AT, Shen FX, Brown JL, Cormack W, Ruiz-Estevez M, Voth JP, et al. The American public is ready to accept human-animal chimera research. Stem Cell Rep. 2020;15:804-10. 
76. Koplin J, Wilkinson D. Moral uncertainty and the farming of human-pig chimeras. J Med Ethics. 2019;45:440-6.

77. Reardon S. First pig-to-human heart transplant: what can scientists learn? Nature. 2022;601:305-6.
Key Words: heart transplantation, surgery, genome editing, blastocyst complementation, xenotransplantation, Fontan operation, bioengeenering 